\title{
Clinical Study \\ Gender-Specific Variation in the Prognosis of Papillary Thyroid Cancer TNM Stages II to IV
}

\author{
Sheng-Hwu Hsieh, ${ }^{1}$ Szu-Tah Chen, ${ }^{1}$ Chuen Hsueh, ${ }^{2}$ Tzu-Chieh Chao, ${ }^{3}$ and Jen-Der Lin ${ }^{1}$ \\ ${ }^{1}$ Division of Endocrinology and Metabolism, Department of Internal Medicine, Chang Gung Memorial Hospital, College of Medicine, \\ Chang Gung University, Linkou 333, Taiwan \\ ${ }^{2}$ Department of Pathology, Chang Gung Memorial Hospital, College of Medicine, Chang Gung University, Linkou 333, Taiwan \\ ${ }^{3}$ Department of General Surgery, Chang Gung Memorial Hospital, College of Medicine, Chang Gung University, Linkou 333, Taiwan
}

Correspondence should be addressed to Jen-Der Lin, einjd@adm.cgmh.org.tw

Received 30 August 2012; Accepted 5 November 2012

Academic Editor: Eleonore Fröhlich

Copyright ( $) 2012$ Sheng-Hwu Hsieh et al. This is an open access article distributed under the Creative Commons Attribution License, which permits unrestricted use, distribution, and reproduction in any medium, provided the original work is properly cited.

\begin{abstract}
To investigate the correlation between gender and the clinical presentation of papillary thyroid cancer and the long-term followup results, 435 patients who underwent total or near-total thyroidectomy were enrolled in this study. Among these papillary thyroid cancer patients, $12.2 \%$ showed lymph node metastases and a higher incidence of male patients in the N1b group. There were 65 from 316 female (20.6\%) and 49 from 120 male (40.8\%) patients who had a postoperative disease progression. A total of $55(12.6 \%)$ patients died of thyroid cancer. Male patients showed a higher thyroid cancer mortality than the females. Multiple regression analysis showed that male gender was an independent risk factor for cancer recurrence and mortality. Male patients with TNM stages II to IV of papillary thyroid cancer need to adopt aggressive surgical and postoperative ${ }^{131}$ I therapy.
\end{abstract}

\section{Introduction}

Thyroid nodules and cancer have long been considered to occur predominantly among women [1]. On the other hand, male gender has been reported to be an important risk factor for the development of the subgroup of welldifferentiated thyroid cancers, based on the occurrence of larger tumors [2], worse prognosis in follicular and Hürthle cell thyroid cancers [3], and lymph node invasion and local neck recurrence of papillary thyroid carcinomas [46]. In contrast, no gender-specific differences have been observed in surgically treatable Graves' disease and toxic thyroid nodules [7]. Thyroid cancer is associated with a wide range of prognoses with different histopathologic patterns. In addition, other factors, such as TNM stage, surgical method, and the application of postoperative adjuvant therapy, may also influence therapeutic outcomes. Even in papillary thyroid carcinoma, different histologic variants with variable presentation and prognoses after treatment have been reported [8].

This study aimed to determine the role of gender in the clinical presentation of papillary thyroid cancer and in the results of long-term followup schemes. To avoid bias due to the influence of age and the less aggressive nature of this cancer in women, papillary thyroid cancers at TNM stage I were excluded from the study. All enrolled patients underwent total thyroidectomy and postoperative remnant ablation. Long-term data were analyzed to identify trends in thyroid cancer progression in relation to gender and age groups.

\section{Patients and Methods}

All patients enrolled in this study underwent total or near-total thyroidectomy and postoperative ${ }^{131}$ I treatment for remnant ablation in Chang Gung Memorial Hospital $(\mathrm{CGMH})$ in Linkou, Taiwan. A total of 435 patients, consisting of 315 women (mean age $56.6 \pm 8.9$ years) and 120 men (mean age $57.9 \pm 9.1$ years), meeting the inclusion criteria during the treatment period between 1986 and 2009 were included in this study. All patients underwent regular followup until the end of 2011. A pathologic review was performed for all thyroid carcinomas using the World Health Organization (WHO) classification [9]. 
After thyroid surgery, thyroid remnant ablation was recommended 4-6 weeks after surgery for patients with papillary thyroid cancers, as in our previous study [10]. The ${ }^{131}$ I ablation dose for most patients was $1.1 \mathrm{GBq}(30 \mathrm{mCi})$. A whole-body scan (WBS) was performed 1 week after ${ }^{131}$ I administration by using a dual-head gamma camera (Dual Genesys, ADAC, USA) equipped with a high-energy collimator. Cases in which the foci of ${ }^{131}$ I uptake extended beyond the thyroid bed were classified as cases of persistent disease or metastases. These patients were given a higher therapeutic dose of $3.7-7.4 \mathrm{GBq}(100-200 \mathrm{mCi}) 3$ to 6 months later. Hospital isolation was arranged for those who received doses exceeding $1.1 \mathrm{GBq}$, and a WBS was performed 2 weeks after administration of the higher therapeutic dose.

All patients were staged according to the UICC-TNM criteria (6th edition) [11]. Patients showing disease progression after the operation were classified into a residual cancer group or a relapse group. Fine-needle aspiration cytology (FNAC), ${ }^{131}$ I WBS, or other noninvasive examination and elevated $\mathrm{Tg}$ levels were used to confirm the presence of local recurrence in the neck or distant metastases. The patients in the residual cancer group were diagnosed within a year of the first thyroidectomy, and those in the relapse group were diagnosed a year after the first thyroid surgery. At the end of 2010, patients were categorized as disease free if they showed negative results in the ${ }^{131} \mathrm{I}$ WBS, undetectable $\mathrm{Tg}$ levels without thyroxine treatment and a TSH level $\geq 30 \mu \mathrm{IU} / \mathrm{mL}$, undetectable Tg antibody levels at the final followup, and no identifiable local or distant metastasis in the noninvasive examination. The study was carried out on humans in compliance with the Helsinki Declaration and following approval by ethics committee of the Institution Review Board in CGMH (reference number: 99-3565B).

All data are expressed as mean \pm SE values. Univariate and multivariate analyses were performed to determine the significance of the various factors by using the Kaplan-Meier method and the log-rank test [12]. A $P$ value $<0.05$ was considered statistically significant. In addition, the survival rates were calculated using the Kaplan-Meier method and compared using the Breslow and Mantel-Cox tests.

\section{Results}

In the 435 subjects with papillary thyroid carcinoma, the mean age of the study population at enrollment was $57.0 \pm$ 9.0 years. Male thyroid cancer patients were generally older than female patients, but no statistical difference was observed $(57.9 \pm 9.1$ years versus $56.6 \pm 8.9$ years; $P=$ 0.1934) (Table 1). Comparison of the mean tumor size in the two groups showed that tumors of a larger size were observed among males. In addition, the incidence of T3 and T4 based on the TNM classification was higher among males. Approximately $12.2 \%$ of the study population demonstrated pathologically proven lymph node metastases. There was a higher incidence of males in the N1b group, although no statistical significance was observed. Higher percentage of males was presented with distant metastases than females (10.8\% versus $4.4 \% ; P=0.0136)$ at the time of diagnosis. Histological presentation at the time of thyroidectomy including TNM stage, multicentric pattern, postoperative $\mathrm{Tg}$, and remnant ${ }^{131} \mathrm{I}$ uptake percentage showed no differences between the genders.

During the followup period, $49(40.8 \%)$ male and 65 (20.6\%) female patients had residual or relapsed. Of this group, $52.6 \%$ (60 of 114) were diagnosed within the first year after thyroidectomy. Higher percentage of residual groups was diagnosed in males ( 28 of $49 ; 57.1 \%$ ) than in females (32 of $65 ; 49.2 \%$ ). Fifty-four patients (33 males and 21 females) had relapsed one year after the operation. The mean duration of relapse after the operation was $4.3 \pm 0.4$ years. A significantly higher relapse frequency was observed among male patients as compared to females $(P=0.0001)$. Approximately $27.8 \%$ of enrolled patients were classified as disease free during followup, with the male group showing a lower frequency than the females, although without statistical significance $(P=0.1267)$. Multiple regression analysis of clinical factors for postoperative progression showed that male gender, postoperative Tg and TNM stage are independent risk factors (Table 2).

After the mean followup period of $7.2 \pm 0.3$ years, 55 $(12.6 \%)$ patients had died of thyroid cancer. The male population showed a higher thyroid cancer mortality than the female population $(24.2 \%$ versus $8.3 \% ; P=0.0001)$. Figure 1(a) shows the cancer-specific survival curves of the male, female, and total groups. The thyroid cancer-specific survival rates in the male, female, and total groups were $86.9 \%, 93.6 \%$, and $92.3 \%$ at 5 years; $72.4 \%, 91.3 \%$, and $86.1 \%$ at 10 years; and $47.2 \%, 71.2 \%$, and $64.3 \%$ at 20 years, respectively. The recurrence-free rates for the male, female, and total groups are $58.9 \%, 81.5 \%$, and $75.3 \%$ at 5 years; $50.1 \%, 73.9 \%$, and $67.6 \%$ at 10 years; and $46.3 \%, 72.7 \%$, and $65.2 \%$ at 20 years, respectively (Figure $1(\mathrm{~b})$ ). In addition, multiple regression analysis showed that male gender and TNM stage are independent risk factors in multiple regression analysis for thyroid cancer specific mortality (Table 3).

\section{Discussion}

The development of thyroid cancer involves multiple stages and genetic mutations, transforming normal follicular epithelial cells to differentiated malignant cancer cells [13]. During the processes of tumor initiation and progression, it has been reported that sex hormones may influence the rates of cancer cell proliferation, migration, or apoptotic change $[14,15]$.

According to prevalence studies, females have a higher incidence rate of thyroid nodules and surgical treatment than males $[16,17]$. In contrast, the incidence of thyroid cancer in nodules is significantly higher in males. Because of differences in screening rates, gender-specific behavior, such as the greater tendency of men to seek medical attention later than females, and surgical methods, the therapeutic outcome varies between genders. [2,6]. Previous study illustrated papillary thyroid cancer in females diagnosed at an earlier age than in males [18]. In contrast, there was no statistical difference of age between genders in this investigation. The main reason was the study exclude the patients in TNM stage I. Age has been used as an important prognostic factor in 
TABLE 1: Clinical features of papillary thyroid carcinoma by gender.

\begin{tabular}{|c|c|c|c|c|}
\hline & Female $(n=315)$ & Male $(n=120)$ & Total $(n=435)$ & $P$ value \\
\hline Age & $56.6 \pm 8.9$ & $57.9 \pm 9.1$ & $57.0 \pm 9.0$ & 0.1934 \\
\hline TNM stage (II/III/IV) & $90 / 60 / 165$ & $27 / 27 / 66$ & $117 / 87 / 231$ & \\
\hline $\mathrm{T} 1 *$ & $128(31.0 \%)$ & $26(19.0 \%)$ & $154(28.0 \%)$ & \\
\hline $\mathrm{T} 2$ & $102(24.7 \%)$ & $35(25.5 \%)$ & $137(24.9 \%)$ & 0.4023 \\
\hline T3 & $39(9.4 \%)$ & $20(14.6 \%)$ & $59(10.7 \%)$ & \\
\hline $\mathrm{T} 4$ & $144(34.9 \%)$ & $56(40.9 \%)$ & $200(36.4)$ & \\
\hline Tumor size $(\mathrm{cm})$ & $3.0 \pm 0.1$ & $3.7 \pm 0.2$ & $3.2 \pm 0.1$ & 0.0001 \\
\hline LN metastases & $38(12.1 \%)$ & $15(12.5 \%)$ & $53(12.2 \%)$ & \\
\hline N1a & $13(41.9 \%)$ & $3(21.4 \%)$ & $16(35.6 \%)$ & 0.1834 \\
\hline N1b & $18(58.1 \%)$ & $11(78.6 \%)$ & $29(64.4 \%)$ & \\
\hline Soft-tissue invasion & $154(48.9 \%)$ & $51(42.5 \%)$ & $205(47.1 \%)$ & 0.2328 \\
\hline Distant metastases & $14(4.4 \%)$ & $13(10.8 \%)$ & $27(6.2 \%)$ & 0.0136 \\
\hline Multicentric & $92(29.2 \%)$ & $30(25.0 \%)$ & $122(28.0 \%)$ & 0.3827 \\
\hline Aggressive histological patterns ${ }^{\#}$ & $8(2.5 \%)$ & $7(5.8 \%)$ & $15(3.4 \%)$ & 0.1366 \\
\hline${ }^{131}$ I dose accumulative dose (mCi) & $152 \pm 10.7$ & $227 \pm 27.9$ & $173 \pm 11.0$ & 0.0023 \\
\hline Postoperative one month ${ }^{131}$ I uptake (\%) & $6.2 \pm 0.8$ & $4.9 \pm 0.7$ & $5.9 \pm 0.6$ & 0.3018 \\
\hline Postoperative progression & $65(20.6 \%)$ & $49(40.8 \%)$ & $114(26.2 \%)$ & 0.0001 \\
\hline Relapse/Residual & $33 / 32$ & $21 / 28$ & $54 / 60$ & 0.4023 \\
\hline Period of relapse from diagnosis (year) & $4.4 \pm 0.7$ & $4.1 \pm 0.6$ & $4.3 \pm 0.4$ & 0.7132 \\
\hline Disease free & $94(29.8 \%)$ & $27(22.5 \%)$ & $121(27.8 \%)$ & 0.1267 \\
\hline Followup period (yr) & $7.3 \pm 0.3$ & $6.8 \pm 0.5$ & $7.2 \pm 0.3$ & 0.3501 \\
\hline Cancer mortality & $26(8.3 \%)$ & $29(24.2 \%)$ & $55(12.6 \%)$ & 0.0001 \\
\hline
\end{tabular}

$\mathrm{T} 1^{*}$ : all the patients with local invasion or distant metastases.

Aggressive histological patterns $s^{\#}$ : tall cell, insular pattern, column cell, and poorly differentiated thyroid cancers.

TABLE 2: Multiple regression analysis for factors associated with postoperative progression.

\begin{tabular}{|c|c|c|c|c|c|c|}
\hline & \multirow{2}{*}{ SE } & \multirow{2}{*}{ Standardized coefficient } & \multirow{2}{*}{$t$ value } & \multirow{2}{*}{$P$ value } & \multicolumn{2}{|c|}{ 95\% confidence interval } \\
\hline & & & & & Lower & Upper \\
\hline Intercept & 0.2438 & -0.677 & -2.724 & 0.007 & -1.167 & -0.186 \\
\hline Age & 0.004 & 0.10 & 1.533 & 0.127 & -0.002 & 0.012 \\
\hline Followup period & 0.005 & -0.049 & -0.711 & 0.478 & -0.014 & 0.006 \\
\hline $\begin{array}{l}\text { Postoperative one month }{ }^{131} \mathrm{I} \\
\text { uptake }\end{array}$ & 0.004 & 0.119 & 1.772 & 0.078 & -0.001 & 0.014 \\
\hline Gender: female/male & 0.068 & 0.251 & 3.672 & 0.000 & 0.115 & 0.383 \\
\hline Postoperative one month Tg & 0.001 & 0.206 & 2.907 & 0.004 & 0.000 & 0.002 \\
\hline TNM stage & 0.035 & 0.175 & 2.576 & 0.011 & 0.021 & 0.157 \\
\hline
\end{tabular}

Dependent variable: postoperative progression (no/yes).

thyroid cancer, and this gender-specific variation thus makes survival analysis between the genders difficult. This study confirmed that based on a specific age group, TNM stage, and therapeutic modality, male gender is associated with a higher relapse rate and cancer-specific mortality.

Most patients with well-differentiated thyroid cancers have undergone long-term followup and treatment with good prognoses. It is important to minimize the risk of recurrence. Male gender has been reported to be an independent risk factor of cancer recurrence $[5,19]$. In this study, consistent surgical and postoperative ${ }^{131}$ I therapy for papillary thyroid cancer in the same institute was associated with a higher recurrence rate. The frequency of male patients in the N1b group was shown to be higher than that of female patients. The incidence of micrometastases in the central neck region in patients staged as N0 by preoperative and intraoperative was recently assessed in papillary thyroid carcinoma [20]. Male gender was shown to be associated with lymph node micrometastasis. In addition, using univariate analysis, a significant correlation was shown 
TABLE 3: Multiple regression analysis for factors associated with cancer mortality.

\begin{tabular}{|c|c|c|c|c|c|c|}
\hline & \multirow{2}{*}{ Standardized coefficient } & \multirow{2}{*}{ SE } & \multirow{2}{*}{$t$ value } & \multirow{2}{*}{$P$ value } & \multicolumn{2}{|c|}{$95 \%$ confidence interval } \\
\hline & & & & & Lower & Upper \\
\hline Intercept & -0.594 & 0.194 & -3.059 & 0.003 & -0.978 & -0.211 \\
\hline Age & 0.104 & 0.003 & 1.405 & 0.162 & -0.002 & 0.009 \\
\hline Followup period & -0.023 & 0.004 & -0.322 & 0.748 & -0.009 & 0.0067 \\
\hline $\begin{array}{l}\text { Postoperative one month }{ }^{131} \mathrm{I} \\
\text { uptake }\end{array}$ & 0.055 & 0.003 & 0.784 & 0.434 & -0.003 & 0.008 \\
\hline Gender: female/male & 0.344 & 0.053 & 4.829 & 0.000 & 0.152 & 0.361 \\
\hline Postoperative one month Tg & -0.051 & 0.001 & -0.688 & 0.434 & 0.000 & 0.001 \\
\hline TNM stage & 0.140 & 0.027 & 1.98 & 0.049 & 0.002 & 0.107 \\
\hline
\end{tabular}

Dependent variable: cancer mortality (yes/no).

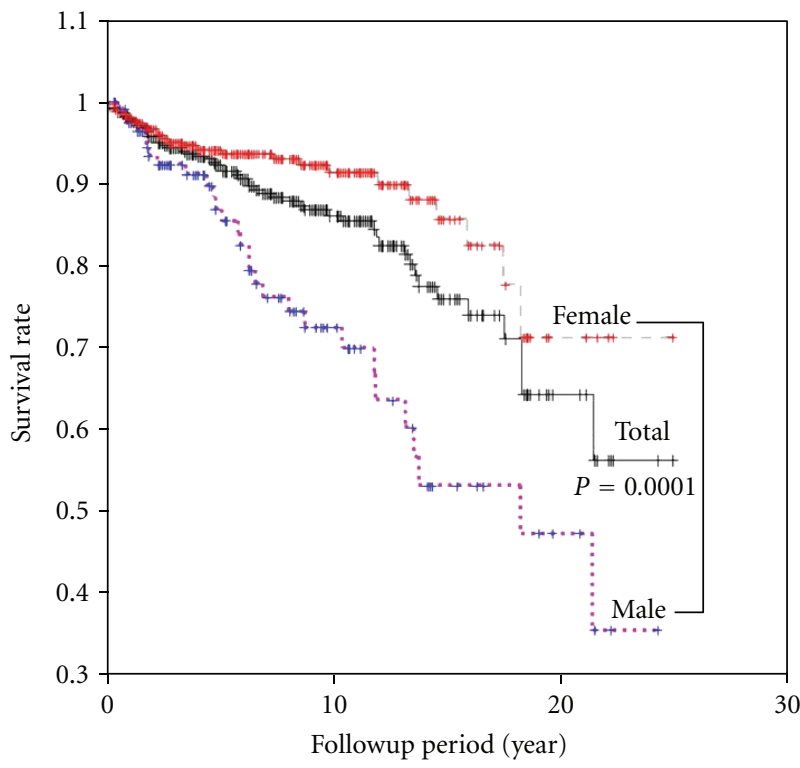

(a)

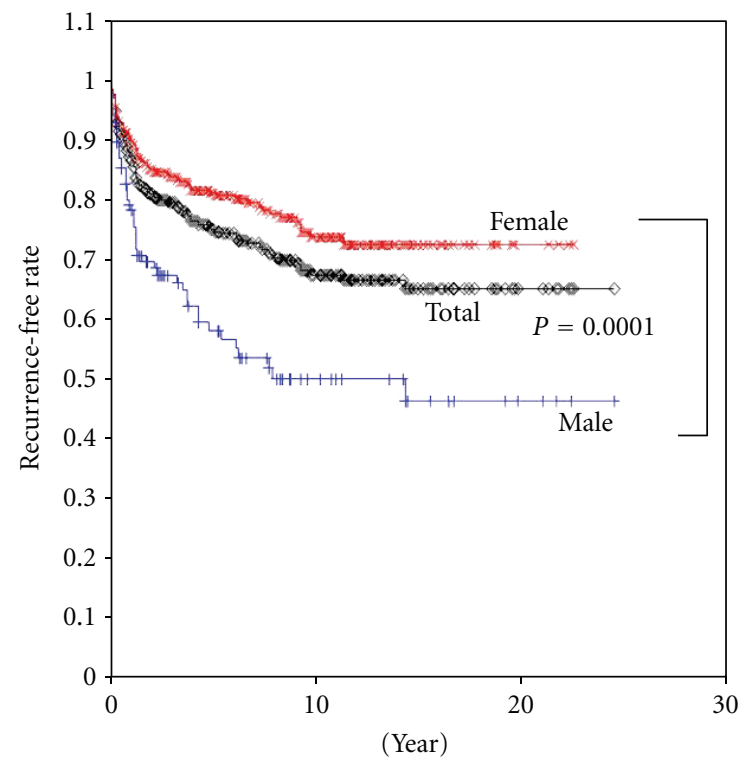

(b)

FIgURE 1: Thyroid cancer mortality (a) and recurrence-free survival (b) curves of the male, female, and total groups.

between male gender and lung metastasis in papillary thyroid carcinoma presenting with bilateral lateral cervical lymph node metastasis [21]. In this study, prophylactic lymph node dissection was not performed on any of the patients. The role of prophylactic lymph node dissection or sentinel node biopsy among male patients at TNM stages II to IV need to be further investigated [22].

Recently published guidelines concerning high-risk cases of papillary and follicular thyroid carcinomas do not consider gender as a risk factor [23, 24]. More aggressive postoperative modalities, such as higher doses of ${ }^{131} \mathrm{I}$ for thyroid remnant ablation and treatment for distant metastases, and closely monitored followup imaging after serum Tg level elevation need to be considered. Those with a histologic pattern of tall cell, insular pattern, and poorly differentiated thyroid cancer have a poorer prognosis, compared with those with classical, well-differentiated thyroid cancer $[25,26]$. Our results illustrated that male patients had higher percentage of aggressive histologic patterns, otherwise no statistical difference. A number of limitations are also identified in this study. The data was selected from a single institution, and this is unlikely to represent the real prevalence of thyroid cancer in Taiwan. This study spanned more than 20 years, with different surgeons performing different surgical procedures. Similarly, the dose used in ${ }^{131}$ I therapy for papillary thyroid cancer varied among endocrinologists. In conclusion, this study demonstrated higher mortality and recurrence rates in males with papillary thyroid cancer in TNM stages II to IV, suggesting the need for more aggressive surgical treatment and postoperative ${ }^{131} \mathrm{I}$ therapy for this specific group of patients.

\section{Conflict of Interests}

There is non-financial competing interest including political, personal, religious, ideological, academic, intellectual, commercial, or any other to declare in relation to this paper. 


\section{References}

[1] M. Brassard, I. Borget, A. Edet-Sanson et al., "Long-term follow-up of patients with papillary and follicular thyroid cancer: a prospective study on 715 patients," Journal of Clinical Endocrinology and Metabolism, vol. 96, no. 5, pp. 1352-1359, 2011.

[2] A. Machens, S. Hauptmann, and H. Dralle, "Disparities between male and female patients with thyroid cancers: sex difference or gender divide?" Clinical Endocrinology, vol. 65, no. 4, pp. 500-505, 2006.

[3] Y. Kushchayeva, Q. Y. Duh, E. Kebebew, A. D’Avanzo, and O. H. Clark, "Comparison of clinical characteristics at diagnosis and during follow-up in 118 patients with Hurthle cell or follicular thyroid cancer," American Journal of Surgery, vol. 195, no. 4, pp. 457-462, 2008.

[4] J. Ricarte-Filho, I. Ganly, M. Rivera et al., "Papillary thyroid carcinomas with cervical lymph node metastases can be stratified into clinically relevant prognostic categories using oncogenic BRAF, the number of nodal metastases, and extranodal extension," Thyroid, vol. 22, no. 6, pp. 575-584, 2012.

[5] T. Nishida, K. Nakao, and T. Hashimoto, "Local control in differentiated thyroid carcinoma with extrathyroidal invasion," American Journal of Surgery, vol. 179, no. 2, pp. 86-91, 2000.

[6] A. Toniato, I. Boschin, D. Casara, R. Mazzarotto, D. Rubello, and M. Pelizzo, "Papillary thyroid carcinoma: factors influencing recurrence and survival," Annals of Surgical Oncology, vol. 15, no. 5, pp. 1518-1522, 2008.

[7] Y. Senyurek Giles, T. Fatih, B. Harika, K. Yersu, T. Tarik, and T. Serdar, "The risk factors for malignancy in surgically treated patients for Graves' disease, toxic multinodular goiter, and toxic adenoma," Surgery, vol. 144, no. 6, pp. 1028-1037, 2008.

[8] R. A. Ghossein, R. Leboeuf, K. N. Patel et al., "Tall cell variant of papillary thyroid carcinoma without extrathyroid extension: biologic behavior and clinical implications," Thyroid, vol. 17, no. 7, pp. 655-661, 2007.

[9] R. A. Delellis, R. V. Lloyd, P. U. Heitx, and C. Eng, "Pathology and genetics of tumors of endocrine organs," in World Health Organization of Tumours, pp. 73-76, IARC, Lyon, France, 2004.

[10] J. D. Lin, K. J. Lin, T. C. Chao, C. Hseuh, and N. M. Tsang, "Therapeutic outcomes of papillary thyroid carcinomas with tumors more advanced than T1N0M0," Radiotherapy and Oncology, vol. 89, no. 1, pp. 97-104, 2008.

[11] L. H. Sobin and C. Wittekind, Eds., TNM Classification of Malignant Tumorsedition, Wiley-Liss, New York, NY, USA, 6th edition, 2002.

[12] D. D. Zhang, X. H. Zhou, D. H. Freeman, and J. L. Freeman, "A non-parametric method for the comparison of partial areas under ROC curves and its application to large health care data sets," Statistics in Medicine, vol. 21, no. 5, pp. 701-715, 2002.

[13] A. De La Chapelle and K. Jazdzewski, "MicroRNAs in thyroid cancer," Journal of Clinical Endocrinology and Metabolism, vol. 96, no. 11, pp. 3326-3336, 2011.

[14] Q. Zeng, G. G. Chen, A. C. Vlantis, G. M. Tse, and C. A. Van Hasselt, "The contributions of oestrogen receptor isoforms to the development of papillary and anaplastic thyroid carcinomas," Journal of Pathology, vol. 214, no. 4, pp. 425-433, 2008.

[15] L. Zhang, R. Rahbari, M. He, and E. Kebebew, "CDC23 regulates cancer cell phenotype and is overexpressed in papillary thyroid cancer," Endocrine-Related Cancer, vol. 18, no. 6, pp. 731-742, 2011.

[16] M. C. Frates, C. B. Benson, P. M. Doubilet et al., "Prevalence and distribution of carcinoma in patients with solitary and multiple thyroid nodules on sonography," Journal of Clinical Endocrinology and Metabolism, vol. 91, no. 9, pp. 3411-3417, 2006.

[17] S. Morganti, G. P. Ceda, M. Saccani et al., "Thyroid disease in the elderly: sex-related differences in clinical expression," Journal of Endocrinological Investigation, vol. 28, no. 11, pp. 101-104, 2005.

[18] J. D. Lin, S. H. Hsieh, H. Y. Chang, C. C. Huang, and T. C. Chao, "Outcome after treatment for papillary thyroid cancer," Head and Neck, vol. 23, no. 2, pp. 140-146, 2001.

[19] A. Jukkola, R. Bloigu, T. Ebeling, P. Salmela, and G. Blanco, "Prognostic factors in differentiated thyroid carcinomas and their implications for current staging classifications," Endocrine-Related Cancer, vol. 11, no. 3, pp. 571-579, 2004.

[20] G. Teixeira, T. Teixeira, F. Gubert, H. Chikota, and R. Tufano, "The incidence of central neck micrometastatic disease in patients with papillary thyroid cancer staged preoperatively and intraoperatively as N0," Surgery, vol. 150, no. 6, pp. 11611167, 2011.

[21] Y. S. Lee, Y. S. Lim, J. C. Lee et al., "Clinical implications of bilateral lateral cervical lymph node metastasis in papillary thyroid cancer: a risk factor for lung metastasis," Annals of Surgical Oncology, vol. 18, no. 12, pp. 3486-3492, 2011.

[22] S. P. Balasubramanian and B. J. Harrison, "Systematic review and meta-analysis of sentinel node biopsy in thyroid cancer," British Journal of Surgery, vol. 98, no. 3, pp. 334-344, 2011.

[23] D. S. Cooper, G. M. Doherty, B. R. Haugen et al., "Revised American thyroid association management guidelines for patients with thyroid nodules and differentiated thyroid cancer," Thyroid, vol. 19, no. 11, pp. 1167-1214, 2009.

[24] F. Pacini, M. Schlumberger, H. Dralle et al., "European consensus for the management of patients with differentiated thyroid carcinoma of the follicular epithelium," European Journal of Endocrinology, vol. 154, no. 6, pp. 787-803, 2006.

[25] A. Machens, H. J. Holzhausen, C. Lautenschläger, and $H$. Dralle, "The tall-cell variant of papillary thyroid carcinoma: a multivariate analysis of clinical risk factors," Langenbeck's Archives of Surgery, vol. 389, no. 4, pp. 278-282, 2004.

[26] J. D. Lin, T. C. Chao, and C. Hsueh, "Clinical characteristics of poorly differentiated thyroid carcinomas compared with those of classical papillary thyroid carcinomas," Clinical Endocrinology, vol. 66, no. 2, pp. 224-228, 2007. 


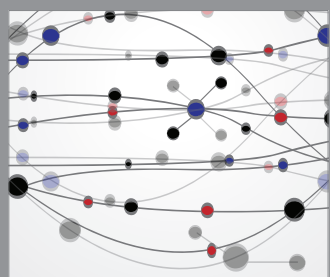

The Scientific World Journal
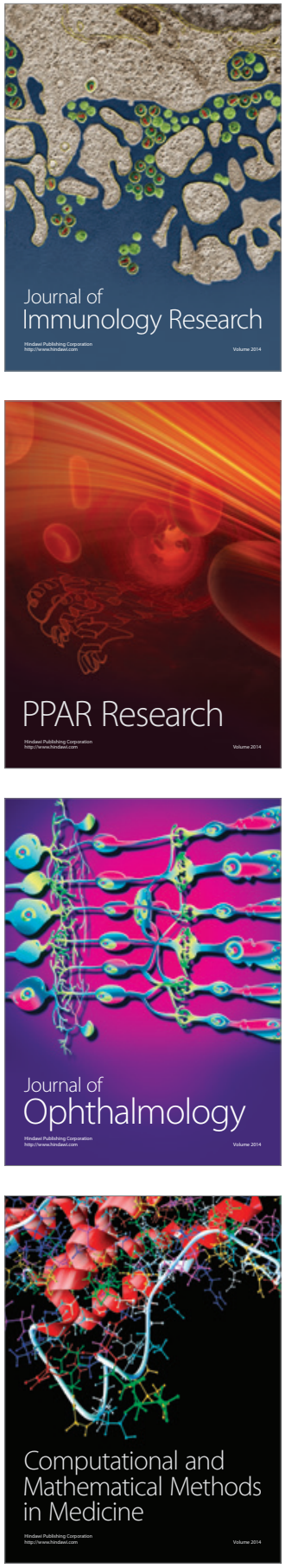

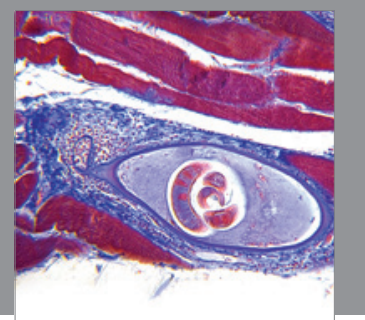

Gastroenterology

Research and Practice
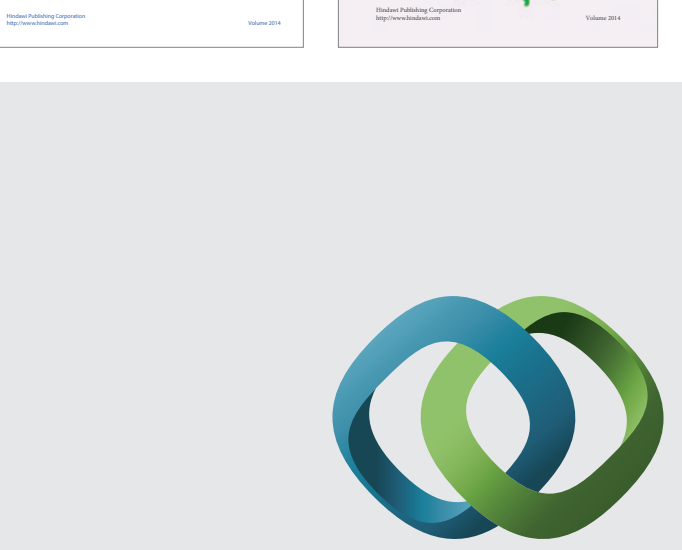

\section{Hindawi}

Submit your manuscripts at

http://www.hindawi.com
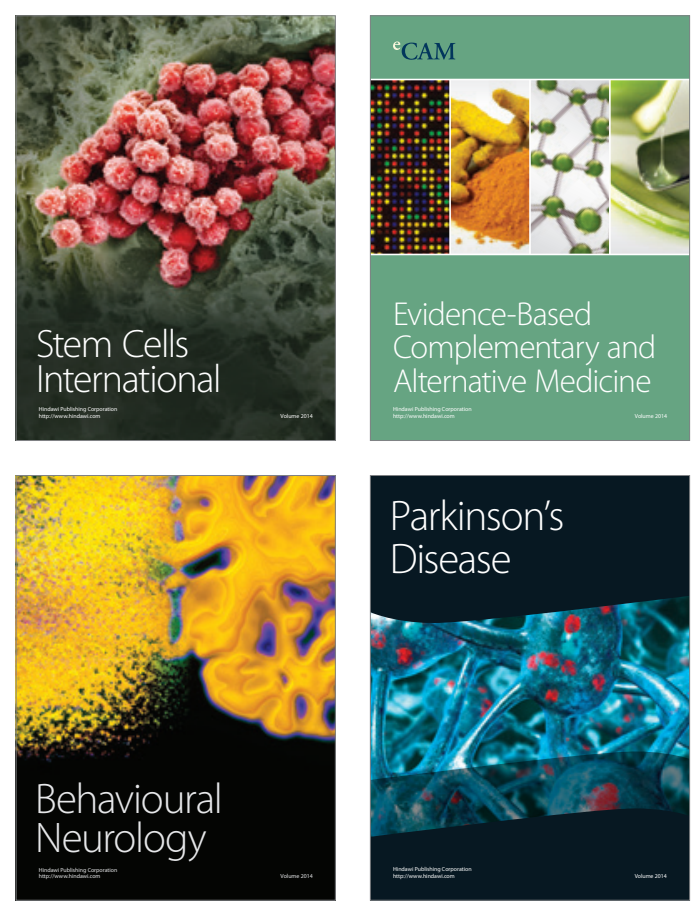

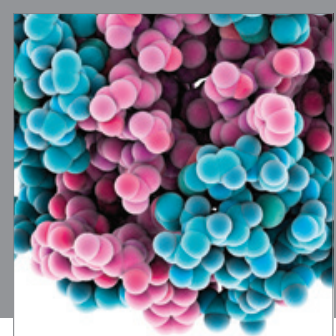

Journal of
Diabetes Research

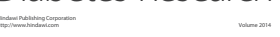

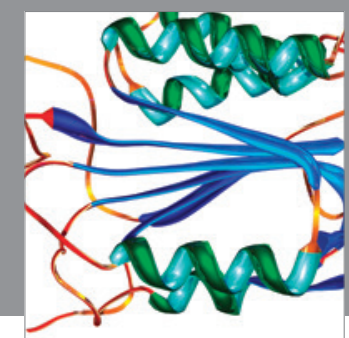

Disease Markers
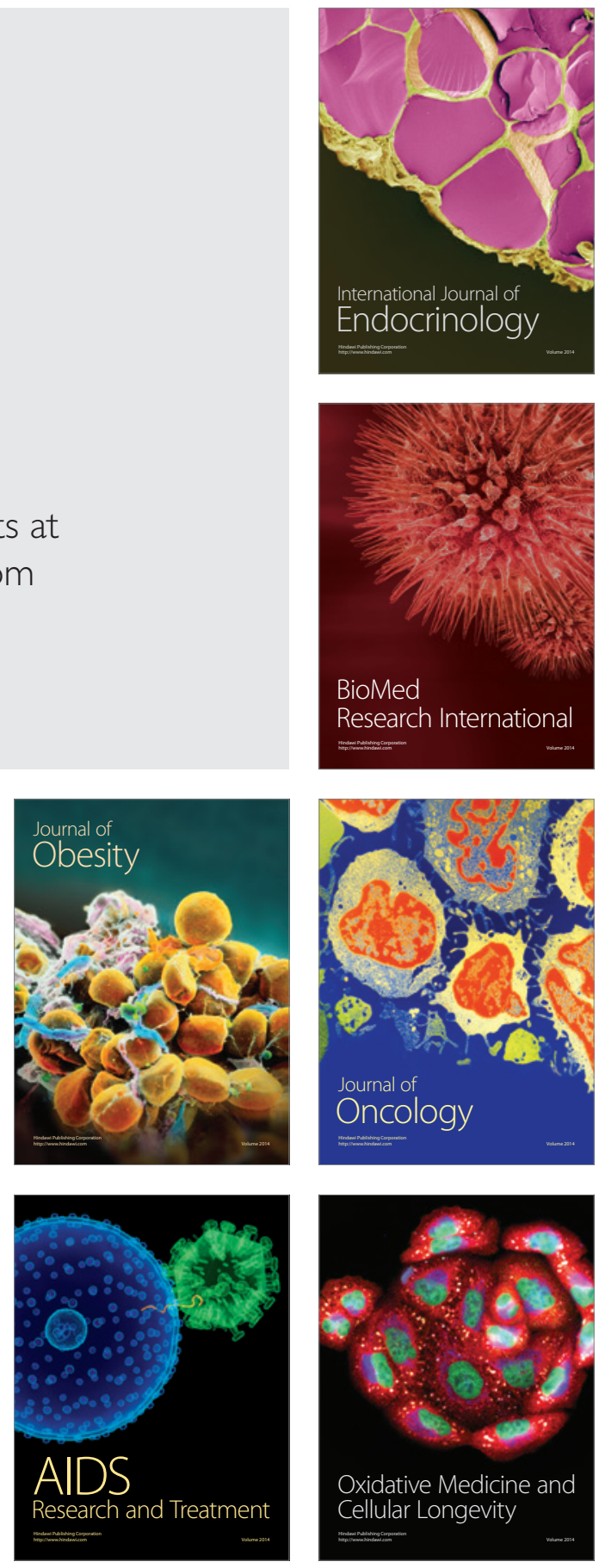